\title{
Una apuesta sustentable en los centros de salud primaria: Una evaluación económica y social
}

\author{
Seguel Sandoval Marco \\ ORCID: https://orcid.org/0000-0002-5022-0138 \\ marcoantonioseguel@gmail.com \\ Universidad del Bío-Bío, Ciudad de Concepción, Chile
}

\author{
Améstica Rivas Luis \\ ORCID: https://orcid.org/0000-0003-0482-0287 \\ lamestica@ubiobio.cl \\ Universidad del Bío-Bío, Ciudad de Concepción Chile
}

\author{
Radrigán Ewoldt Rudi \\ ORCID: https://orcid.org/0000-0002-4181-9325 \\ rradriga@udec.cl \\ Universidad de Concepción, Ciudad de Concepción Chile
}

Recibido (29/04/21), Aceptado (14/05/21)

Resumen: El objetivo de este trabajo es evaluar un proyecto fotovoltaico como fuente de energía alternativa en el sector de salud primaria como estudio de caso, desde la perspectiva económica y social. La evaluación se basó en variables técnicas y económicas bajo los criterios de Valor Actual Neto (VAN) y Tasa interna de retorno (TIR), valorizando las reducciones de carbono (CO2) y utilizando la tasa de descuento social del Ministerio de Desarrollo Social. Los resultados son favorables y sugieren la ejecución de este proyecto como iniciativa de política pública. Sin embargo, queda en evidencia que en periodos de invierno no se cubre las necesidades energéticas, haciendo imprescindible diversificar la matriz con fuentes tradicionales.

Palabras Clave: energía solar fotovoltaica, sector salud, sustentabilidad, evaluación social.

\section{Sustainable bet in primary health centers. An economic and social evaluation}

\begin{abstract}
The aim of this work is to evaluate a photovoltaic project as an alternative energy source in the primary health sector as a case study, from the economic and social perspective. The evaluation was based on technical and economic variables under the criteria of Net Present Value (NPV) and Internal Rate of Return (IRR), valuing the carbon reductions (CO2) and using the social discount rate of the Ministry of Social Development. The results are favorable and suggest the implementation of this project as a public policy initiative. However, it is clear that in winter periods energy needs are not covered, making it essential to diversify the matrix with traditional sources.
\end{abstract}

Keywords: Fotovoltaic solar energy, health sector, sustainability, social evaluation

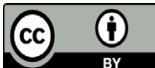




\section{Introducción}

La energía es un insumo clave para el desarrollo de los países y cuando esta se obtiene y utiliza de manera óptima incide en el crecimiento económico y ofrece oportunidades para el cuidado del medio ambiente, permitiendo así avanzar hacia un desarrollo sustentable. La sustentabilidad es un imperativo para todo gobierno, tanto para los países desarrollados como los emergentes. En este sentido, Chile ha establecido uno de los ejes prioritarios en su agenda energética, a través del fomento y competencia en el mercado de generación distribuida y autoconsumo, posibilitando a través de instituciones estatales de distintos sectores un aporte significativo a la sustentabilidad energética. En tanto, el sector salud en Chile es uno de los con mayor importancia social, ya que atiende al $80 \%$ de la población [1]. Por ello, la experiencia en la incorporación de nuevas tecnologías energéticas en este sector, especialmente la energía solar fotovoltaica en sus dependencias podría servir de referente como experiencia base para la implementación de políticas pública.

Es sabido, que el sector energía es responsable en gran medida de contribuir al cambio climático. Los procesos de producción y uso de combustibles fósiles como fuente de energía generan los llamados gases de efecto invernadero (GEI), principalmente dióxido de carbono (CO2) entre otros gases [2]. Una de las mayores fuentes de contaminantes de dióxido de carbono ( $\mathrm{CO} 2)$ son las plantas de generación de electricidad . Estudios demuestran que por 1 kilowatts hora (Kwh) de consumo de electricidad en un hogar que es generado por una planta termoeléctrica, se emiten 0.7 kilo gramos de dióxido de carbono (CO2/gr) [3]. Chile es un contribuyente menor en emisiones de gases de efecto invernadero (GEI), representa un $0.2 \%$ del total global [4]. Sin embargo, según el último estudio nacional oficial de GEI, las emisiones del sector energético han aumentado significativamente en las últimas décadas. Estas muestran que al año 2018 el sector industrial Energía, en el que se encuentra inserto la generación de electricidad, transporte y calefacción, representa el $78 \%$ de las emisiones totales, seguido por Agricultura $(10,8 \%)$, Procesos industriales y uso de productos $(6,2 \%)$ y Residuos (5,2\%) [5] .

Por otra parte, el aumento de la población y el desarrollo de los países emergentes, cada día demandan más energía, por tanto el consumo energético global es creciente, sobre todo el de la energía eléctrica, ocasionando cambios en el ámbito de las redes de alimentación eléctricas, así como también en la renovación y aplicación de las centrales eléctricas [6]. Hay que mencionar además, cuando se hacen estimaciones de los costos asociados a las externalidades negativas, producidas por las fuentes de generación que usan combustibles fósiles, no es tan evidente en terminos comparativos que las energías renovables sigan siendo más caras. En efecto, dicho costo dependerá también de otros factores, como la valoración que se haga de los impactos en salud, calidad de vida y en el medio ambiente.

En este contexto, en la investigación se analiza la rentabilidad bajo un enfoque económico social, incorporando la valorización de las emisiones de carbono como un beneficio social al implementar energía solar fotovoltaica (SFV) en las techumbres de unidades de salud en Chile, con el caso de los centros de salud familiar (CESFAM) en la región de Nuble. Para ello se analizó las variables de costos de implementación del SFV y los beneficios de la generación de electricidad para autoconsumo.

\section{Desarrollo}

\section{A.Importancia de la energía sustentable en el mundo}

Se estima que la energía solar recibida cada diez días sobre la tierra equivale a todas las reservas conocidas de petróleo, gas y carbón. Por este motivo, la energía solar fotovoltaica (SFV) es una de las soluciones que más intereses ha despertado en los nuevos proyectos de generación de electricidad a nivel mundial. Considerada a su vez como factor clave y estratégico para alcanzar las metas de desarrollo económico y social de los países, por lo que es indispensable asegurar el suministro energético futuro en concordancia con las exigencias que la sociedad impone a este sector. En el proceso de conversión de energía SFV en energía eléctrica se tiene en cuenta la radiación que llega a la tierra, llamada irradiancia y es definida como, potencia por unidad de área normal a la dirección de propagación, y esta expresada en watt por metro cuadrado (w/m2), de esta forma la conversión se realiza a través de celdas fotovoltaicas, conformándose así los denominados generadores fotovoltaicos [7].

Los países latinoamericanos son energéticamente dependientes y esto a su vez refuerza la necesidad de diversificar sus economías y buscar sustitutos energéticos. Es por este motivo que han puesto atención y han incrementado significativamente su participación en el mercado de las energías renovables, especialmente en la SFV [8], donde países como México, Brasil, Argentina y Chile lideran la incorporación de este tipo de energía. La SFV marca la nueva tendencia de generación de electricidad, y ha sido catalogada como uno de los principales insumos para la mitigación de la pobreza y aumento del desarrollo socioeconómico de los países. Sin embargo, aún existe controversia respecto a que la energía total generada por un sistema fotovoltaico, no compensa la energía consumida durante su fabricación, considerado como un proceso intensivo desde el punto de vista energético [9]. Ahora bien, si se mira desde el punto de vista del tiempo de recuperación de la energía que genera una panel solar, y se considera el ciclo de vida completo de la generación fotovoltaica, desde la minería del cuarzo hasta su disposición final, la energía consumida es solamente una fracción de la energía generada [10]. De manera análoga, investigaciones han comprobado que la energía consumida en los procesos del ciclo de vida de los paneles solares se compensan entre un promedio de tres años [11], siendo a su vez mas eficientes, porque generan en el mismo lugar que se consume, atenuando las pérdidas por distribución.

Por otra parte, el recurso solar tiene un rol fundamental en la mitigación de los GEI, por ser renovable. Asimismo la 
energía solar fotovoltaica constituye una fuente de energía limpia e inagotable desde el punto de vista del consumo, lo que garantiza que no se agote y que se considere en principio limpia o verde, porque contaminan muy poco y no emiten GEI. [11].

Por tanto, la Unión Europea (UE) ha establecido que las Energías Renovables No Convencionales (ERNC) son las soluciones que los países deben de establecer a un corto y mediano plazo. En esta línea, en Dinamarca un $43 \%$ de la energía producida es renovable y la meta es llegar a un $70 \%$ en el 2020 , Alemania cuenta con un porcentaje del $25 \%$ y como objetivo alcanzar un $80 \%$ en 2050 . Asimismo, China a pesar de los numerosos desafíos, es la principal fuente mundial de inversiones en energía renovable, además de ser el mayor fabricante de paneles solares fotovoltaicos, convirtiendola en el epicentro de la revolución mundial en energía limpia, sobrepasando a otras regiones [12].

\section{B.Implementación de iniciativas}

Esta innovadora y sostenible idea de energización para el entorno de la edificación se ha expandido en todo el mundo, así los SFV presentan buenos rendimientos operacionales. Ejemplo de ello, es el caso de Nigeria, que con una capacidad media diaria de generación eléctrica de 3.810 megawatt hora (Mwh) inicialmente pasaron a generar 91.419 (Mwh) de energía eléctrica diaria a partir del 2015 [13]. Otro caso de éxito es Egipto, sus proyectos han generado rentabilidad positiva, aprovechando su gran índice de irradiancia, buenas condiciones del clima, esto ha incentivado a invertir en la construcción de SFV integrados [14] con el fin de proporcionar a las edificaciones un flujo energético lo más constante posible o para ayudar a los edificios a satisfacer parcialmente la demanda energética.

Otro caso relevante es Alemania con la ley de preferencia a las energías renovables desde el 2000, con la implantación de techos solares, lo cual permiten que este país tenga el 30\% de la capacidad instalada de generación fotovoltaica mundial [15], permitiendo que los hogares accedan al servicio energético al ser instalados en los techos y fachadas, integrándose arquitectónicamente en el diseño propio de edificios y viviendas. Así, las energía renovable, no tan solo se basan en criterios ecológicos y aporte medioambientales, también por motivo de autónomia que las energías renovables poseen.

C.Energías renovables no convencionales, experiencias locales

Chile desde el año 2008 cuenta con la Ley 20.257 de energías renovables no convencionales (ERNC) [2], que promueve la generación de energía eléctrica. Para ello se eligió el sistema de cuotas, mediante el cual se establece la obligación a las empresas eléctricas que un porcentaje de la energía que comercializan provenga de fuentes ERNC, incentivando el desarrollo de este tipo de proyectos, mejorando sus condiciones de acceso al mercado y otorgando exenciones de peajes por transmisión. Los resultados demuestran que la capacidad solar fotovoltaica instalada ha aumentado de casi cero desde el año 2008 a más de 1.6 Gigawatt $(\mathrm{Gw})$ de potencia eléctrica instalada al 2017 [16]. Posteriormente, en 2014 se promulga la Ley 20.571, llamada Ley de Generación Distribuida como se indica en distintos documentos [2], [17]; la cual regula el pago de las tarifas eléctricas de las generadoras residenciales y permite la autogeneración de energía en base a ERNC y cogeneración eficiente. Esta ley, entrega el derecho a los usuarios a vender sus excedentes directamente a la distribuidora eléctrica a un precio regulado, el cual está publicado en el sitio web de cada empresa distribuidora, cuyo límite de generación actual de 300 Kilowatts (KW). Al analizar al 2019 las instalaciones acogidas a Ley 20.571, se puede identificar que la energía solar es la más ampliamente desarrollada en el país con $18.171 \mathrm{Kw}$ de potencia instalada $(99,39 \%)$, seguida por biomasa, cogeneración eficiente e hidarúlica con proyectos marginales.

Todos estos instrumentos han logrado que Chile desplace las barreras y estimule la incorporación de las ERNC a la matriz de generación eléctrica nacional, como una forma de aportar a los objetivos de la política energética chilena, Es más, los esfuerzos del Estado de Chile, ha desarrollado un incremento de proyectos de ERNC, que actualmente aportan a la matriz energética un $20.9 \%$ al total [18]. En esta misma línea, se ha implementado el programa techos solares públicos [19], que es una iniciativa del Ministerio de Energía inserta en la agenda de energía, orientado a instalar sistemas fotovoltaicos en los techos de los edificios públicos, representando una solución innovadora y sostenible para el entorno de la edificación, representando un esfuerzo del sector público. De esta forma se espera así poder contribuir a la maduración del mercado.

\section{D.Energías alternativas y sustentabilidad en recintos de sa- lud}

Se destaca la experiencia en hospitales de Alemania donde se concluyó que al integrar nuevas tecnologías de energía y elementos de eficiencia energética en áreas como iluminación, calefacción, aire acondicionado y calentamiento de agua, se logra un ahorro entre el $25 \%$ y el $40 \%$ en el gasto [20]. Es evidente que los recintos asistenciales demandan una gran cantidad de energía asociada a calefacción, iluminación e equipamiento eléctrico, aportando en forma indirecta emisiones de GEI por el alto consumo de energía para su funcionamiento. Ejemplo de ello, es un estudio sobre la huella de carbono en Argentina en el hospital general de agudos Enrique Tornú de Buenos Aires en el año 2015, donde se demostró que la huella de carbono anual fue de 1526.47 toneladas de dióxido de carbono equivalente (ton $\mathrm{CO} 2 \mathrm{eq} /$ año), siendo el área de energía el responsable de aportar con $29 \%$ de emisiones indirectas. Por tanto, la emisiones del área energía representan una externalidad negativa. Los aportes de esta experiencia hospitalaria, permiten identificar las fuentes de emisión de mayor impacto, como áreas de oportunidad para la implementación de estrategias de reducción y/o mitigación [21].

En el Reino Unido, se calcula que los hospitales repre- 
sentan el 25\% del total de las emisiones del sector público, en tanto las actividades de los hospitales representan entre el $3 \%$ y el $8 \%$ de la huella del cambio climático en países desarrollados como Inglaterra y los Estados Unidos [22]. En este sentido, las fuentes de energías alternativas, específicamente la solar fotovoltaica integrada en edificios de atención de salud, es un aporte relevante a la sustentabilidad, asi lo demuestran las experiencias internacionales desarrolladas en hospitales de Alemania, España, Estados Unidos y Japón, definen el uso de energía alternativas como casos exitosos, donde los gobiernos han tomado protagonismo en incentivar el uso de energías renovables, además de incentivar el mercado al establecer como instrumento principal el incentivo energético feed-in tariff. Este incentivo es una forma de subvencionar las energías renovables mientras éstas no puedan competir con otras fuentes energéticas establecidas.

Los hospitales son esenciales para la sociedad, pero también pueden causar daños al medio ambiente, de esta forma al integrar energía solar fotovoltaica se convierte en una alternativa para mitigar el daño, y se considera una fuente de energía limpia. Es más, las publicaciones de sustentabilidad en recintos de salud se limitan al manejo de los residuos sólidos hospitalarios y se descuidan otros aspectos como el consumo de energía.

Un estudio de caso local, el que tuvo como propósito identificar y plantear una metodología integral experimentada en el pabellón de hospitalización del hospital clínico del sur de Concepción Chile. Este trabajo permitió detectar potencialidades de la envolvente y evaluar tres distribuciones combinadas de equipos térmicos y fotovoltaicos en la cubierta del edificio, determinando una alternativa con una cantidad de 131 paneles térmicos y 196 paneles fotovoltaicos que puede satisfacer hasta un $70 \%$ del suministro de agua caliente sanitaria y un $91 \%$ de la iluminación, recuperando la inversión inicial en un periodo de 7 a 8 años [23]. Ahora si bien, en Chile se han desarrollado varios proyectos de incorporación de energía solares fotovoltaica en el sector salud y las experiencias demuestran buenos resultados tanto en la rentabilidad económica como en el cuidado del medio ambiente. Sin embargo, algunos estudios abordan aspectos técnicos de manera acotada y discusiones en términos generales de la importancia de este tipo de tecnología, omitiendo las externalidades medioambientales y sociales.

\section{Metodología}

Para analizar la incorporación de energía solar fotovoltaica (SFV) en los techos de unidades de salud llamadas Centro de salud familiar (CESFAM) en Ñuble, Chile, se realizó un análisis de rentabilidad bajo un criterio privado y luego de carácter social, relevando la importnacia en la disminución de los GEI. Metodológicamente, la valoración de los costos y beneficios se realizó a través de precios de mercado, y dado que se propone una evaluación social se hizo necesario realizar ajustes para reflejar un costo más aproximado del valor para la sociedad, por tanto se descontó el impuesto al valor agregado (IVA).
A nivel de costeo, se valoró tanto la incorporación de energía SFV instalada en el techo del CESFAM, como el beneficio social por ahorro de costos en energía eléctrica que supone el reemplazo por el sistema fotovoltaico destinado a autoconsumo. Además, se consideró eel beneficio de valorizar las emisiones de dióxido de carbono $\mathrm{CO} 2$.

En tanto, los costos incluyen la inversión, reinversión y manteniniento del SFV, que está relacionada con la cantidad de paneles solares, equipamiento eléctrico, infraestructura y mano de obra necesaria para la instalación sobre la superficie del techo del CESFAM. Para su determinación se utilizó como base las estadísticas de la estructura de costos de los proyectos licitados, insertas en el estudio de reporte de costos de licitación públicas entre los años 2015-2018. Asimismo, se procedió a utilizar la herramienta explorador solar del Ministerio de Energía, para realizar una evaluación preliminar del potencial energético y trazar la superficie donde serán instalados los paneles solares [22]. En este mismo sentido, el explorador solar presenta la información pública más detallada que existe actualmente sobre el recurso solar en Chile, y permite estimar la superficie en metros cuadrados (m2) como el comportamiento del ciclo anual de energía eléctrica generada por el SFV en megawatt hora mensual (Mwh/m).

Para determinar los beneficio económicos generados, se calcula la energía eléctrica generada anualmente por el sistema de 196.140 kilowatt hora al año (Kwh/a), multiplicada por el precio social de la energía de la empresa distribuidora de la zona de concesión. Para el caso de estudio, se determina un precio CLP\$ 89 el kilowatt hora (Kw/h) [24], al cual se le descuenta el 19\% del impuesto al valor agregado (IVA), resultando un precio social de la energía en CLP\$ 75.

Asimismo, la reducción de $\mathrm{CO} 2$ equivalente se considera un beneficio para el proyecto y es valorizada considerando el precio social del carbono establecido por el Ministerio de Desarrollo Social (MDS) [25] a CLP\$ 19.995 la tonelada de $\mathrm{CO} 2$ equivalente. Este precio se multiplica por la cantidad de emisiones que se logran disminuir con la incorporación de energía que aporta el SFV. Las emisiones reducidas, es el producto de la energía anual generada por el SFV 196.140 kilowatt hora al año (Kwh/a) por el factor se emisión del sistema eléctrico nacional es de 0.397 este factor indica la cantidad de kilogramos de $\mathrm{CO} 2$ equivalente por cada kilowatt hora consumido $(\mathrm{kgCO} 2 \mathrm{eq} / \mathrm{kWh})$. Por tanto, la valoración del $\mathrm{CO} 2$ es el producto de, las emisiones reducidas por el precio social del carbono del (MDS). Esta valorización representa cuanto estaría dispuesto a pagar la sociedad hoy para evitar un daño futuro causado por una tonelada de carbono adicional emitida al medio ambiente, coherente con el acuerdo de Paris.

Posteriormente, cuantificados los costos y beneficios, se constuye la proyeccion de rentabilidad y se considera un flujo de caja propuesto por el Ministerio de Desarrollo Social, que cosidera una tasa de descuento de $6 \%$, con un horizonte de evaluación al año 20 , considerando el valor residual de los paneles fotovoltaicos y la estructura necesaria para su instalación. Esta valoración se hace de acuerdo a las estimaciones 
de los fabricantes, quienes establecen una vida útil promedio de 25 años, este valor se calcula como el valor presente de los flujos de beneficios netos entre el año 21 y 25 , considerando la reinversión del equipamiento inversor electrónico, que es necesario para que el sistema siga funcionando.

Para comprobar la viabilidad del proyecto se utilizó la metodología de evaluación financiera de proyectos de inversión propuesta por Sapag (2007) [2], a través de los indicadores de rentabilidad Valor Actual Neto (VAN) y Tasa Interna de Retorno (TIR), considerando una tasa de descuento propuesta por el Ministerio de Desarrollo Social (2018) de un 6\%. Finalmente para contrastar los datos de generación de energía aportada por el SFV y el consumo de energía demandado por los CESFAM, se utilizó como referencia el perfil de consumo eléctrico del actual proyecto en construcción en la Región de Ñuble, en la comuna de Coihueco, estos datos se solicitaron a la Dirección del Servicio de Salud de Ñuble.

\section{Resultados}

A.Características del Sistema fotovoltaico

A través de una herramienta denominada explorador solar se estimó la generación de electricidad del sistema fotovoltaico, considerando los niveles de irradiancia y las condiciones meteorológicas en el sitio de interés. Para el caso de estudio se definió la ubicación en la Región de Ñuble, específicamente en la comuna de Coihueco, donde actualmente se esta construyendo el nuevo proyecto CESFAM de la comuna. El sistema calculado para el sitio donde se construye el CESFAM se indica en la Tabla I, entrega una potencia eléctrica máxima de 137.46 kilowatt pico (Kwp) que es la máxima potencia que genera el conjunto de paneles solares en las horas de máxima de insolación solar, la generación promedio de energía diaria es de 581 kilowatt hora $(\mathrm{Kwh})$, la generación promedio de energía mensual es de 17.70 Megawatt hora (Mwh) y como resultado anual el SFV genera 211.96 Megawatt hora (Mwh) de energía.

TABLA 1. Generación eléctrica fotovoltaica

\begin{tabular}{|l|c|c|}
\hline \multicolumn{1}{|c|}{ Características del SFV } & Datos & Tipo de unidad \\
\hline Potencia instalada & 137.46 & $\mathrm{kwp}$ \\
\hline Generación de energía diaria & 581 & $\mathrm{kw} / \mathrm{hora}$ \\
\hline Generación de energía mensual & 17.70 & $\mathrm{Mw} / \mathrm{hora}$ \\
\hline Generación de energía anual & 211.96 & $\mathrm{Mw} / \mathrm{hora}$ \\
\hline Superficie utilizada & 1.800 & $\mathrm{~m} 2$ \\
\hline
\end{tabular}

Fuente: elaboración propia.

\section{B.Ciclo anual de generación fotovoltaica}

El SFV presenta su mayor nivel de generación de electricidad entre los meses de septiembre a abril, siendo el mes de enero donde se presenta la mayor cantidad de energía aportada de 24.48 Megawatt hora (Mwh), los niveles más altos de generación de energía están relacionado con el incremento en los niveles de irradiancia que se presenta en estos meses por las buena condiciones climáticas en la zona. Por otra parte, se aprecia una considerable disminución de energía del 50\% entre los meses de mayo a agosto, el nivel más bajo se presenta en el mes de junio con 10.45 Megawatt hora (Mwh), si bien podemos apreciar niveles de energía bajos en los meses más críticos, el sistema no pierde capacidad de generación eléctrica, por tanto aun cuando el sistema está en el rendimiento más bajo de generación, este puede seguir entregando electricidad.

C.Costos de inversión, reinversión y mantenimiento del SFV

Para la determinación de los costos se utiliza como base las estadísticas de la estructura de costos de los proyectos licitados, insertas en el estudio de reporte de costos de licitación públicas entre los años 2015-2018 de techos solares públicos del Ministerio de Energía de Chile.

Por tanto la inversión del SFV se determina multiplicando la potencia eléctrica instalada del SFV 137.46 kilowatt pico (Kwp) indicada en Tabla I, por el precio promedio ofertado en licitación pública de la región del Bío Bío, siendo la más cercana a la región de Ñuble. Las estadísticas del estudio indican que el precio por cada 1 watt equivale a CLP\$ 782 (USD\$1.15), por tanto la inversión del proyecto tiene un 
costo de CLP\$ 107.493.720 (USD\$ 158.079), ahora para la evaluación del proyecto con enfoque social, esta Inversión se traduce a precio social y se obtiene descontando un 19\% correspondiente al impuesto al valor agregado (IVA), resultando en una inversión de CLP\$90.330.857 (USD\$ 132.839).

La reinversión se ingresa en el año $\mathrm{t}=10$ del flujo de caja y corresponde al costo del equipo eléctrico inversor que tiene una vida útil de 10 años, este tiene un valor de CLP\$ 10.934.319.

Los costos de mantenimiento anuales se estiman en base a un precio social referencial de CLP\$ 1.519.363, implican limpieza, mano de obra, insumos, obtenido del análisis de precios de mantenimiento efectuado por el Ministerio de Energía (2015). Para determinar el valor residual del sistema fotovoltaico se basó en el estudio de Cisterna, Améstica-Rivas y Piderit (2020) [2], tomó en cuenta las estimaciones de los fabricantes que establecen una vida útil promedio de 25 años, por tanto el valor residual se estima como el valor presente de los flujos de beneficios netos entre los año $t=20$... $\mathrm{t}=25$, y considerando la reinversión del equipo eléctrico inversor, resultando un valor residual de CLP\$ 42.311.845, este valor se ingresa en el flujo de caja en el año $t=20$.

\section{D.Beneficios de la incorporación del SFV}

La valoración de la energía generada por el sistema fotovoltaico se considera como uno de los beneficios, y se obtiene a partir de la energía eléctrica generada anualmente por los paneles solares multiplicada por el precio social de la energía de la empresa distribuidora de la zona de concesión, para este caso corresponde a la empresa CGE distribución. Por tanto, el SFV genera anualmente un promedio de 196.140 kilowatt hora al año (Kwh/a), y el precio social del kilowatt hora $(\mathrm{Kw} / \mathrm{h})$ de la empresa de distribución, tiene un precio de CLP\$ 89, para traducir este precio a social se debe descontar el 19\% del impuesto al valor agregado (IVA), resultando un precio social de la energía en CLP\$ 75, por tanto la valoración anual de la energía generada es CLP\$ 14.710.500.

Al incorporar energía SFV, se logra la disminución de $\mathrm{CO} 2$ equivalente, por tanto se considera un beneficio valorado. El precio social del carbono establecido por el ministerio de desarrollo social, es de CLP\$ 19.995 la tonelada de $\mathrm{CO} 2$ equivalente, este precio se multiplica por la cantidad de emisiones que se logran disminuir con la incorporación de generación de energía que aporta el SFV. Las emisiones reducidas, es el producto de la energía anual generada por el SFV 196.140 kilowatt hora al año (Kwh/año) por el factor se emisión del sistema eléctrico nacional es de 0.397, resultando 78 toneladas de $\mathrm{CO} 2$ equivalentes que se logran reducir y valorizada en CLP\$ 1.556.966.

\section{E.Flujo de caja y proyección de costo beneficio}

Al realizar la evaluación económica del proyecto, en la Tabla II se proyecta el comportamiento de generación eléctrica del SFV en operación, donde se puede observar dos vías de ingreso económicos, la primera es la valoración promedio de energía anual generada por los paneles solares, el segundo ingreso está conformado por la valoración promedio de disminución anual de toneladas de $\mathrm{CO} 2$ equivalente, se utiliza para valorar los aumentos o disminuciones por tonelada de $\mathrm{CO} 2$ en la evaluación social de proyectos de inversión, cuando se compare la cantidad de emisiones de $\mathrm{CO} 2$ de la situación base con las emisiones de las distintas alternativas de proyecto, por tanto a la evaluación del proyecto se le agrega una dimensión ambiental y social, que al utilizar energía sustentables presentan ventaja sobre proyectos de energía tradicionales. En otra línea, se encuentran los costos anuales de operación y mantenimiento al SFV. Como resultado se suman los dos ingresos menos los costos, los que consolidan el flujo anual resultante.

TABLA 2. Proyección del Beneficio anual

\begin{tabular}{|c|c|c|c|}
\hline $\begin{array}{c}\text { Valoración promedio } \\
\text { energía anual generada } \\
\text { por SFV CLP\$ }\end{array}$ & $\begin{array}{c}\text { Valoración promedio } \\
\text { Disminución anual del } \\
\text { CO2 equivalente CLP\$ }\end{array}$ & $\begin{array}{c}\text { Promedio anual de } \\
\text { operación y } \\
\text { mantenimiento CLP\$ }\end{array}$ & $\begin{array}{c}\text { Flujo anual } \\
\text { promedio resultante } \\
\text { CLP\$ }\end{array}$ \\
\hline$\$ 13.515 .564$ & $\$ 1.556 .966$ & $-\$ 1.443 .395$ & $\$ 15.198 .011$ \\
\hline
\end{tabular}

Fuente: elaboración propia.

\section{F.Rentabilidad Social}

Teniendo en cuenta, los datos de costo beneficio expuestos en la tabla anterior, se construyó la proyección de rentabilidad del proyecto de incorporación de energía SFV al CESFAM. De acuerdo con los datos resultantes del flujo de caja observados en la Tabla III, los indicadores de rentabilidad Valor Actual Neto (VAN) y Tasa Interna de Retorno (TIR), resultan ambos positivas, bajo estos resultados se puede inferir que es rentable realizar este tipo de proyecto en forma pública. 
TABLA 3. Rentabilidad Social del proyecto

\begin{tabular}{|c|c|c|c|c|}
\hline $\begin{array}{c}\text { Inversión } \\
\text { inicial CLP\$ }\end{array}$ & $\begin{array}{c}\text { Re-inversión } \mathrm{t}=\mathbf{1 0} \\
\text { CLP\$ }\end{array}$ & $\begin{array}{c}\text { Valor desecho } \\
\text { económico } \mathbf{t}=\mathbf{2 0} \text { CLP\$ }\end{array}$ & VAN Social CLP\$ & $\begin{array}{c}\text { TIR } \\
\text { Social \% }\end{array}$ \\
\hline$\$ 91.119 .328$ & $\$ 10.934 .319$ & $\$ 42.311 .845$ & $\$ 74.500 .043$ & $14 \%$ \\
\hline
\end{tabular}

Fuente: elaboración propia.

G.Aporte de energía eléctrica del SFV y demanda del CESFAM

La Figura 1, muestra el contraste de los datos, de la generación de electricidad del SFV con la demanda de energía del CESFAM, se puede apreciar que durante los meses de mayor presencia de irradiancia. Es decir, entre los meses de noviembre a marzo, donde el SFV puede entregar energía para su autoconsumo proporcional a la totalidad demandada por el recinto durante el mes. Por otro lado, durante los meses de marzo a octubre el SFV no es capaz de generar la demanda energética total de las instalaciones. Si bien, no es suficiente para dotar de energía en su totalidad, el sistema aporta energía en términos económico y reduce los efectos ambientales.

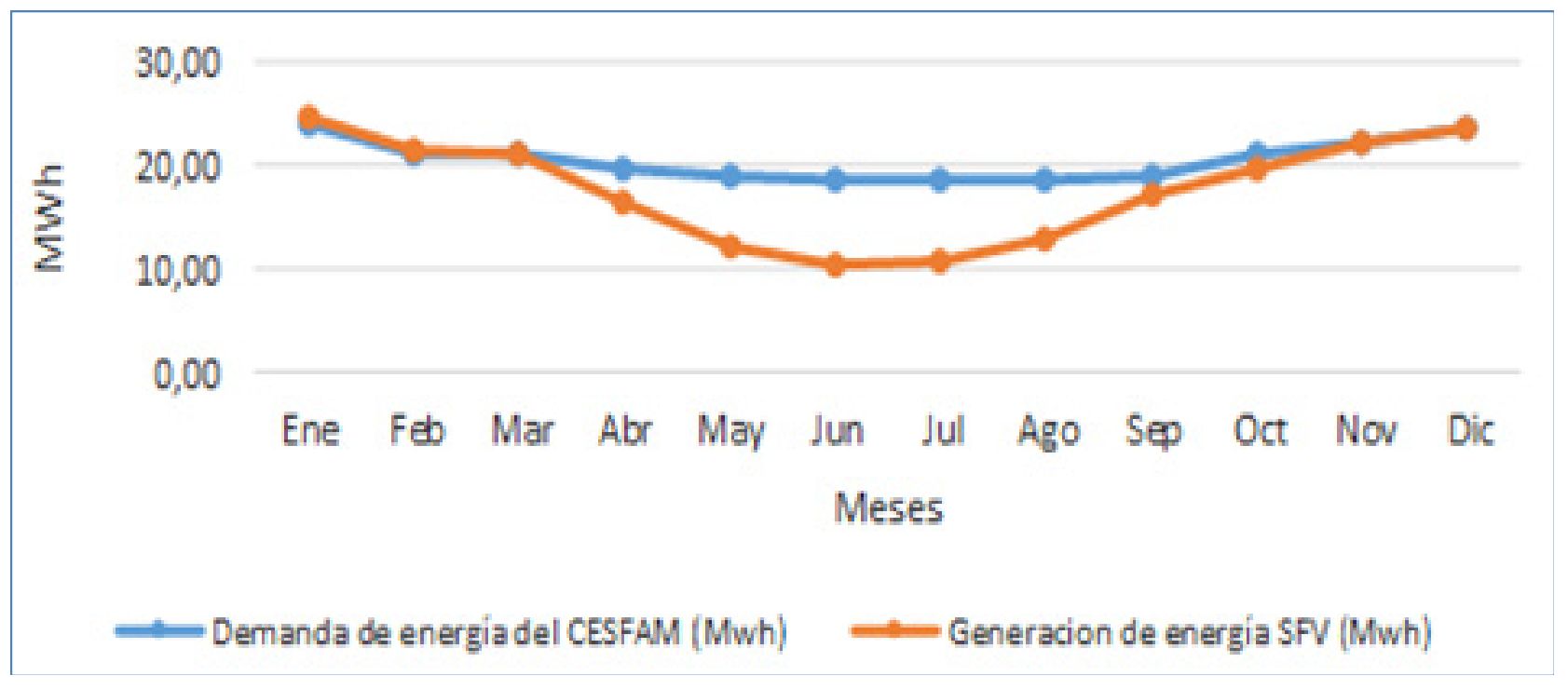

Fig 1. Generación del SFV versus Demanda del CESFAM

Los indicadores de rentabilidad económicos demuestran que el proyecto de incorporación de energía SFV a centros de salud primaria, bajo una perspectiva social, tienen una rentabilidad positiva tanto el VAN como la TIR. Estos resultados positivos pueden ser explicados por el tipo de enfoque costo beneficio adoptado, además que se consideró en la evaluación del proyecto una tasa de descuento social del $6 \%$ establecida por el ministerio de desarrollo social, además de los ingresos como beneficio social, por ahorro de costos en energía eléctrica reemplazada por el sistema fotovoltaico que se destina a autoconsumo, esta valorización representa una vía de ingreso importante, el segundo benéfico que se considera ingreso, es la valorización de la reducción de emisiones de carbono equivalente, que se consigue al incorporar este tipo de energía baja en emisiones.

Por otro lado, si bien el costo de la inversión inicial del proyecto es alto, al considerar los costos de las ultimas licitaciones realizadas en el país y que en cierta medida ha contribuido a madurar y a desarrollar un nuevo mercado de empresas especialistas en este tipo de tecnología, se han logrado economías de escalas al licitar proyectos que implican un tamaño mayor y mayor cantidad de actores en las ofertas, haciendo una notoria disminución de los precios ofertados en licitaciones públicas desde el año 2015 a la fecha de hoy.

Desde un punto de vista de operación del SFV se observó que la generación de electricidad entre los meses de marzo a octubre, no alcanza a cubrir la demanda total del centro de salud, este comportamiento del sistema puede ser de base para 
un estudio posterior, pero una hipótesis que se puede inferir es que durante los meses de menor generación de energía, ocurre a causa de niveles bajos de irradiancia solar, por el cambio de estación climática. En este sentido, para el caso de estudio se hace necesario que el CESFAM mantenga la actual fuente de energía, conectada a la red de distribución de la compañía de electricidad, por tanto en los periodos o meses de déficit energético que el SFV no pueda abastecer de electricidad la demanda total, la fuente tradicional mantenga el servicio normal de energía eléctrica. Por otro lado, en relación con las emisiones, se observó que existe una reducción significativa de las emisiones de carbono 78 toneladas anuales (tonCO2/año) al utilizar este tipo de tecnología SFV.

\section{Conclusiones}

Las energías renovables representan un factor clave y estratégico para las economías emergentes. En este sentido, Chile cuenta con un potencial para el desarrollo de energías renovables no convencionales como la fotovoltaica, donde el sector público no podría estar ajeno. En este sentido, el año 2014 a través del Ministerio de Energía del Gobierno de Chile se crea el programa de techos solares en edificios públicos.

Sin embargo, evaluar la conveniencia económica y social de estos proyectos es un desafío permanente y complejo, especialmente en territorios alejados de las grandes urbes. El sector salud, dada su importancia estratégica, posee instalaciones a lo largo de todo el país, siendo los centros de salud pública, unidades con alto potencial para desarrollar dichas iniciativas. Aunque la ejecución de proyectos fotovoltaicos en este sector aún es una alternativa que pasa por consideraciones políticas y económicas de los tomadorse de decisiones.

En este trabajo se evidencia la conveniencia económica de llevar acabo este tipo de proyecto, donde los beneficios que aporta el SFV son mayores a los costos involucrados, contribuyendo al ahorro de energía y permitiendo el autoconsumo como la reducción de las emisiones de GEI. No obstante, la incorporación del SFV no cubre en su totalidad la demanda energética del centro de salud, situación que se genera en los meses de menos irradiancia solar. Por tanto, la incorporación de este tipo de tecnología debe considerarse como una contribución al autoconsumo parcializado de electricidad, que trabaja de forma complementaria al sistema tradicional.

En virtud de la demanda de energía en el sector salud, las inversiones futuras en infraestructura deberían contemplar las energías alternativas como una opción económicamente más viable, aprovechando las economías de escala que se llevan acabo. Destaca el caso de sectores sin acceso a electricidad donde las fuentes de energías alternativas pueden abastecer a establecimientos de atención primaria de la salud ubicados en lugares más remotos, siendo a su vez soluciones técnicas menos vulnerables en un país caracterizado por catástrofes naturales y alta dependencia de los sistemas tradicionales basados en combustibles fósiles.

Es importante enfatizar que si bien se realizó una evaluación económica con una valorización de la reducción de emi- siones de carbono, aportando una visión social y medioambiental de los proyectos; esta es una dimensión del tema que puede tener otras pesrpectivas de análisis. Asimismo, la experiencia analizada desde una mirada local, no necesariamente es extrapolable a otras realidades, dado los costos y accesos a las tecnologías como a condiciones ambientales favorables para la ejecución de proyectos SFV.

\section{Referencias}

[1]Fondo Nacional de Salud (FONASA), Boletin Estadístico 2016-2017. Disponible: https://www.fonasa.cl/sites/fonasa/ adjuntos/Boletin_Estadistico_2016_2017_2018

[2]Cisterna L, Améstica-Rivas L, Piderit M. Proyectos fotovoltaicos en generación distribuida ¿Rentabilidad privada $\mathrm{o}$ sustentabilidad ambiental?. Revista Politécnica. 2020; 45(2): en prensa. Disponible:

https://revistapolitecnica.epn.edu.ec/ojs2/index.php/revista_politecnica2/issue/view/39

[3]Medina J. La dieta de dióxido de carbono CO2. Conciencia Tecnológica. 2010; 39: 50-53. Disponible: https://www. redalyc.org/articulo.oa?id=94415753009

[4]Mardones C. Muñoz, T. Impuesto al CO2 en el sector eléctrico chileno: efectividad y efectos macroeconómicos. Economía Chilena. 2017; 20(1): 4-25. Disponible: https://www. bcentral.cl/web/guest/articulos-publicados

[5]Ministerio del Medio Ambiente, Tercer Informe de Actualización Bienal de Chile, 2018. Disponible: https://mma.gob. cl/wp-content/uploads/2019/07/2018_NIR_CL.pdf

[6]Gallego Y, Arias R, Casas L, Sosa R. Análisis de la implementación de un parque fotovoltaico en la Universidad Central de las Villas. Ingeniería Energética, 2018; 39(2): 8290. Disponible: http://rie.cujae.edu.cu/index.php/RIE/article/ view/531.

[7]Arias R, Pérez I. Nueva metodología para determinar los parámetros de un módulo fotovoltaico. Ingeniería Energética. 2018; 39(1): 38-47. Disponible: http://rie.cujae.edu.cu/index. php/RIE/article/view/557

[8]Plá J, Bolzi C, Durán J.C. Energía Solar Fotovoltaica. Generación Distribuida conectada a la red. Ciencia e Investigación. 2018; 68(1), 51-64. Disponible: http://aargentinapciencias.org/wp-content/uploads/2018/03/tomo68-1/4-Duran-cei68-1-5.pdf

[9]Hou G, Sun H, Jiang Z, Pan Z, Wang Y, Zhang X, Zhao Y, Yao Q. Life cycle assessment of grid-connected photovoltaic power generation from crystalline silicon solar modules in China. Applied Energy. 2016; 164 (15): 882-890. Disponible: https://doi.org/10.1016/j.apenergy.2015.11.023

[10]Baharwani V, Meena N, Dubey A, Brighu U, Mathur J. Life Cycle Analysis of Solar PV System: A Review. International Journal of Environmental Research and Development. 2014; 4(2): 183-190. Disponible: https://www.ripublication. com/ijerd_spl/ijerdv4n2spl_14.pdf

[11]Rojas-Hernández I, Lizana F. Tiempo de recuperación de la energía para sistemas fotovoltaicos basados en silicio cristalino en Costa Rica. Ingeniería Energética. 2018; 39 (3): 
195-202. Disponible: http://rie.cujae.edu.cu/index.php/RIE/ article/view/544

[12]World Economic Forum. Informe Energía. 2017. Disponible: https://es.weforum.org/agenda

[13]Zou L, Wang L, Lin A, Zhu H., Peng Y, Zhao Z. Estimation of global solar radiation using an artificial neural network based on an interpolation technique in southeast China. Journal of Atmospheric and Solar-Terrestrial Physics. 2016; 146: 110-122 Disponible: https://doi.org/10.1016/j. jastp.2016.05.013

[14]Crawley D, Lawrie, L, Winkelmann F, Buhl W, Huang C, Pedersend C, Strand R, Liesen R, Fisher D, Witte M, Glazer J. EnergyPlus: creating a new-generation building energy simulation program. Energy and Buildings. 2001; 33(4): 319-331. Disponible: https://doi.org/10.1016/S0378-7788(00)00114-6 [15]Larrain S, Stevens C, Paz M. Las fuentes renovables de energía y el uso eficiente. 2002. LOM Ediciones, Chile Disponible: http://www.archivochile.com/Chile_actual/patag_sin_repre/03/chact_hidroay-3\%2000010.pdf

[16]World Economic Forum. Cuatro países que lideran las tendencias de energía solar en América Latina y el Caribe, 2017.Disponible: https://es.weforum.org/agenda/2017/05/ cuatro-paises-que-lideran-las-tendencias-de-energia-solar-en-america-latina-y-el-caribe/

[17]Ministerio de Energía. Ley 20.571, Regula el pago de las tarifas eléctricas de las generadoras residenciales. 2012. Disponible: https://www.leychile.cl/Navegar?idNor$\mathrm{ma}=1038211$.

[18]Comisón Nacional de Energía (CNE) de Chile. Reporte mensual sector energético. 2019; 50. Disponible: https:// www.cne.cl

[19]Ministerio de Energía, Programa de Techos Solares Públicos, Reporte de costos. 2018. Disponible: http:/www. minenergia.cl/techossolares/wp-content/uploads/2017/04/ Reporte-de-Costos-de-Adjudicacion-2018-233x300.jpg.

[20]Löhr W, Gauer K, Serrano N, Zamorano A. Igarss 2014. Eficiencia Energética en Hospitales Públicos. Editorial GTZ - Dalkia. Santiago de Chile.

[21]Smith M, De Titto E. Hospitales sostenibles frente al cambio climático: huella de carbono de un hospital público de la ciudad de Buenos Aires. Revista Argentina Salud Pública. 2018; 9(36): 7-13. Disponible: http://rasp.msal.gov.ar/ rasp/articulos/volumen36/7-13.pdf

[22]Chung J, Meltzer, D. Estimate of the carbon footprint of the US health care sector. Jama. 2009; 302(18):1970-1972. Disponible: https://jamanetwork.com/journals/jama/article-abstract/184856

[23]Nope A, García R, Bobadilla A. Método para la implementación de sistemas solares activos en establecimientos hospitalarios, estudio de caso en el hospital clínico del sur, Concepción, Chile. En Proceedings of the 3rd International Congress on Sustainable Construction and Eco-Efficient Solutions. Sevilla. 2017; 451-464. Disponible: https://idus. us.es/xmlui/handle/11441/58969

[24]Compañía General de Electricidad, Tarifa de Suministro. 2018 Disponible: http://www.cge.cl/wp-content/
uploads/2019/08/Publicacion-CGE-2019-08-01-Suministro-electrico.pdf

[25]Ministerio de Desarrollo Social, Precio Social del Carbono. 2018. Disponible: http://sni.ministeriodesarrollosocial. gob.cl/download/precio-social-co2-2017/?wpdmdl=2406

\section{RESUMEN CURRICULAR}

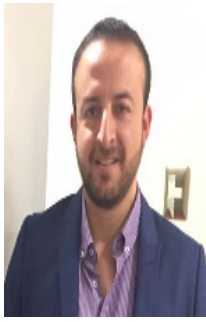

Marco Seguel Sandoval, Magíster en Gestión de Empresas, Departamento de Gestión Empresarial, Facultad de Ciencias Empresariales, Universidad del Bío Bío, Chile.

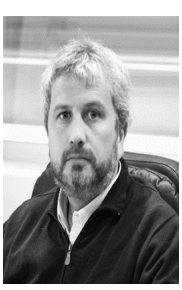

Luis Améstica-Rivas (*),Dr. en Administración y Dirección de Empresas. Académico Departamento de Gestión Empresarial, Facultad de Ciencias Empresariales, Universidad del Bío-Bío, Chile. Investigador asociado grupo de investigación en Dirección Universitaria de la Universidad Politécnica de Cataluña (UPC), España

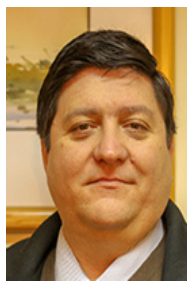

Rudi Radrigán Ewoldt, Dr. en Sistemas e Ingeniería en Máquinas Eléctricas, Electrónicas y de Control. Académico de la Facultad de Ingeniería Agrícola de la Universidad de Concepción, Centro de Desarrollo Tecnológico Agroindustrial (CDTA) UdeC, Chile. 